\title{
LAS ESTRUCTURAS DE COMUNICACIÓN HIDRÁULICA EN LA VEGA ALTA DEL SEGURA (EDAD MODERNA) Y SU APORTE AL TURISMO
}

\author{
Juan Carlos Trigueros Molina
}

Universidad de Murcia

\section{RESUMEN}

Este estudio pretende analizar las estructuras comunicativas de carácter hidráulico (Puentes, cunas y barcos) durante la Edad Moderna en la Vega Alta del Segura (Región de Murcia). La finalidad es reconocer la identidad turística de este patrimonio hidráulico. Con el objeto de llegar a este propósito ha sido necesario examinar con detalle dos líneas de temática: la histórica y el paisaje natural y cultural.

Palabras clave: puentes históricos; cultura turística; Vega Alta del Segura; paisaje natural; vías de comunicación hidráulica.

Hydraulic communication structures in the Vega Alta del Segura and its contribution to tourism (Modern Age)

\section{ABSTRACT}

The present study aims to analyze the communication structures of a hydraulic nature (bridges, cradles and boats) during the Modern Age in the Vega Alta del Segura (Region of Murcia). The purpose is to recognize these units for their tourist identity. To reach this conclusion it has been necessary to examine in detail two lines of topics: the historical and the natural and cultural landscape.

Keywords: historical bridges; tourist culture; Vega Alta del Segura; natural landscape; water communication roads.

Fecha de recepción: 16 de febrero de 2017.

Fecha de aceptación: 19 de julio de 2017.

E-mail: juancarlos.trigueros@um.es 


\section{INTRODUCCIÓN}

Existe un agente esencial para que la sociedad tenga la oportunidad de practicar un turismo global: los medios que facilitan el transporte de un lado a otro. Es probable que sin vías de comunicación el recreo de viajar estuviera estancado y casi paralizado. Este panorama afectaría negativamente al bloque socio-económico y comercial. El presente estudio no pretende examinar la articulación y los tipos de vías que permiten el desplazamiento de vehículos para propósitos de ocio, ya fueran de una índole acuática, aérea o terrestre. Se centra en una interpretación tanto del pasado (Edad Moderna) como del presente de las principales y más antiguas estructuras comunicativas de carácter hidráulico (puentes, cunas y barcos) en la Vega Alta del Segura (municipios de Cieza, Abarán y Blanca), Región de Murcia, con el fin de reconocer su identidad turística.

No obstante, el avance, en esta ocasión, no va yuxtapuesto a instalaciones, reformas con nuevos equipamientos, plataformas o a las innovadas tecnologías de la información y la comunicación, que, como se reconoce, han abierto un escaparate de visibilidad, tanto en los ámbitos naturales, urbanos, como, asimismo, en entornos más rurales, como la huerta (García y Canales, 2017). Más bien, el desarrollo pretendido está decantado a otro sector: el conocimiento. Es tan importante el papel que juega el patrimonio no renovable y las estrategias económicas a fines al turismo, como, igualmente, el sentido y las referencias informativas relativas al monumento natural o cultural. Como manifestó, Ramón y Cajal, en Tónicos de la voluntad, “ Una nueva verdad constituye la ventura más grande a que puede aspirar el hombre”; y, es que, los acontecimientos históricos por descubrir pueden causar impresiones emocionales e impactos morales, cognitivos de manera individual y colectiva. En efecto, lo que se alude es a un turismo basado en la experiencia.

Como sabemos, la curiosidad y el saber incentivan a la sociedad a viajar de una vertiente a otra. El conocimiento es un componente base y endógeno del turismo. Por ello, el argumento de este trabajo se inclina más hacia la línea cultura turística, que hacia un turismo cultural; en otras palabras, se apoya en el progreso del conocimiento con el fin de ser un medio de mejora cuando la actividad informativa sea ejercida por un guía; o, sencillamente, para hacer posible una tarea turística. Lo que aportamos, por una parte, se trata de un progreso correspondiente a la divulgación ilustrativa a través de la investigación humanística sobre estos complejos. Este factor es tan importante como cualquier otro para las empresas pertenecientes a distritos turísticos culturales, ya que puede resultar atrayente para un público, no solo especializado, sino que busque experiencias de una misma índole por el territorio español y europeo.

Por otra parte, hay que añadir que el presente estudio también sirve para organizar todo el contenido referente a estos bienes inmuebles. El guía o el medio emisor tienen la obligación de exponer el contenido informativo de una manera estructural y didáctica. Por estas razones, es necesario realizar una revisión sobre aquellas estructuras comunicativas de carácter hidráulico y de mayor valor histórico en el área de investigación. Por supuesto, al mismo tiempo se intenta comprobar si el conjunto de datos recolectados por medio de diferentes bibliografías tienen la posibilidad de renovar y enriquecer sus testimonios históricos por medio de un enfoque heurístico tradicional: la búsqueda de fuentes documentales inéditas. Además, se señala la belleza del entorno natural donde 
están ubicados. El ambiente, las vistas paisajísticas también juega un papel llamativo y sugestivo para que estas unidades se identifiquen como elementos turísticos, al ser bienes accesibles tanto día como de noche (Véase Figura 1). Por tanto, se encuentran dos campos esenciales: la historia y el paisaje. Como aliciente se sugiere un itinerario turístico que puede interesar al público.

Para alcanzar los objetivos históricos ha sido necesario enfocar en primer lugar la investigación en dos laboratorios principales: el Archivo Histórico Provincial de Murcia, acudiendo a Protocolos Notariales referentes a Cieza, Abarán y Blanca; y los Archivos Municipales de los citados municipios y sus respectivas Actas Capitulares. Todos los datos extraídos se han contrastado con aquellos provenientes de las bibliografías. Al mismo tiempo, hay que señalar el hallazgo de una variedad documental. Destacan los testamentos, las ordenanzas, los acuerdos, los convenios y los pleitos. Correspondiente al paisaje ha sido interpretado desde un punto ambiental, resaltando los detalles más llamativos; y, por último, ofrecemos como culmen del trabajo un reducido plan turístico.

\section{Figura 1 \\ IMAGEN NOCTURNA DEL PUENTE VIEJO DE ABARÁN}

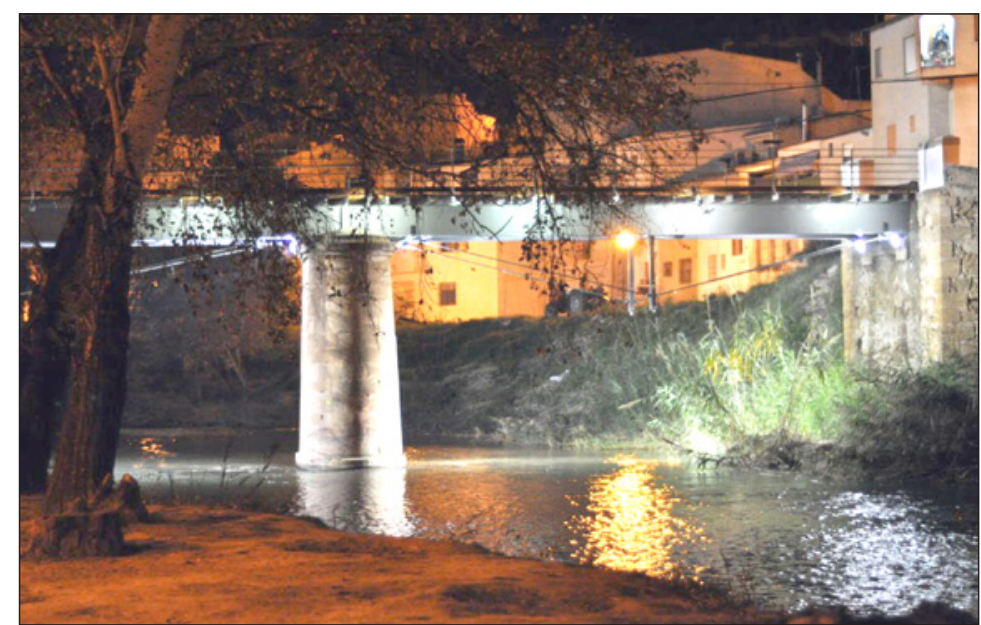

Fuente: Imagen optenida junto al Parque Municipal de Abarán (Fotógrafo J.C. Trigueros, 10/09/2017)

Ahora bien, el primer apartado, analiza las monografías y la situación actual de las vías de comunicación, encuadrando los enclaves históricos donde se ha centrado el estudio. A continuación, se muestra una tabla con los datos principales. Seguidamente, se organizan dos bloques generales que parten de los datos de la Tabla: el primero dedicado a las vías de comunicación rurales (cunas y barcos) y el segundo centrado en puentes de madera y de piedra. En ellos se interpretan y se comparan la información recogida. Posteriormente, se presenta las características más primordiales del paisaje y, seguidamente, como es compresible, el estudio se remata con unas conclusiones, donde se comenta algunas estrategias para incentivar el turismo de estos bienes inmuebles. 


\section{RECONOCIMIENTO DE LA ACTUALIDAD}

Como sabemos los puentes han sido arquitecturas de ingeniera amoldadas su evolución a la utilidad de nuevas materias primas y técnicas para su diseño, edificación y mantenimiento. Esto se ha debido por las tendencias progresistas de la ingeniería profesional, por la revolución tecnológica y por la demanda de la sociedad. Actualmente, existe en Cieza cuatro puentes, unos más rudimentario que otros, en Abarán otros cuatro, y en Blanca uno (Véase Figura $2^{1}$ y Tabla 1). De los nueve puentes, tres son los analizados por el motivo de instalarse en puntos clave e históricos (Puente de los Nueve Ojos, en Cieza; Puente Viejo en Abarán, y Puente de Hierro en Blanca); es decir, sitios donde se han edificado en tiempo atrás otras vías de comunicación de carácter más preindustrial.

Estos apuntes se han extraído por medio de tres fuentes: monografías, fotografías históricas y declaración de BIC de un solo puente. Respecto a las monografías sobresale por su excelencia documental y metodológica Hierro, piedra y madera: los puentes ciezanos sobre el Segura (Siglo XVI-XX), compuesta por Alfredo Marín Cano (2010), acerca de los puentes y cunas utilizadas por los ciezanos para pasar el Segura. Igualmente permanecen dos referencias monográficas acerca del Puente Viejo de Abarán, en un cuadro temporal de los siglos XIX y XX. El primero constituido por el cronista oficial de Abarán, José David Molina Templado en $1980^{2}$. Su contenido informativo ayudó a que este inmueble fuera nombrado BIC con categoría de Monumento por la Dirección General de Bienes Culturales, dependiente de la Consejería de Cultura y Portavocía de la Región de Murcia en 2006. Por otra parte, Felix Martínez García se basa en una similar línea que el autor anteriormente citado, aunque analiza de forma más técnica las diferentes estructuras del puente (Martínez, 2010). Estos autores ofrecen datos de un periodo más antiguo referentes a vías de comunicación más rústicas en este punto clave: las cunas. Por tanto, no se señala la construcción de un puente de piedra durante la Edad Moderna. Anexado al puente se encuentra un cartel informativo (Figura 2) que indica algunos datos generales del citado bien. Todo esto conduce a pensar de la no existencia de otros puentes de madera o de piedra en fechas más tempranas que las mencionadas. Por último, sobre el pasado de los puentes en Blanca se desconoce la existencia de estudios específicos vinculados con tal temática.

Una concepción hay que tener clara: la ausencia de vestigios históricos en referencia a modelos estilísticos del cuerpo del puente. Ello supone un desconocimiento sobre si estas fábricas comunicativas del área de investigación reflejaron en su composición las dos tendencias arquitectónicas surgidas durante el siglo XVII en el reino castellano: una conservadora y otra innovadora. La conservadora estuvo encabezada por Tomas Ramón, el Hermano Bautista (Correla, 1985; Barrio, 1986) o Fray Lorenzo de Nicolás (López, 1990; Díaz, 2008; Fernández, 1994; Sánchez y Roda, 2001). Estos arquitectos e ingenieros practicaron unas líneas técnicas y tácticas coincidentes: viaductos largos, con gran número de ojos desiguales, con luces muy pequeñas y un gran número de ojos desiguales

1 http://sitmurcia.es/visor/? Visto: 05-01-2017

2 http://www.boe.es/buscar/doc.php?id=BOE-A-2006-3141 Visto: 12-12-2016. 


\section{Figura 2 \\ VISTA AÉREA DE CIEZA, ABARÁN Y BLANCA. UBICACIÓN DE SUS PUENTES}
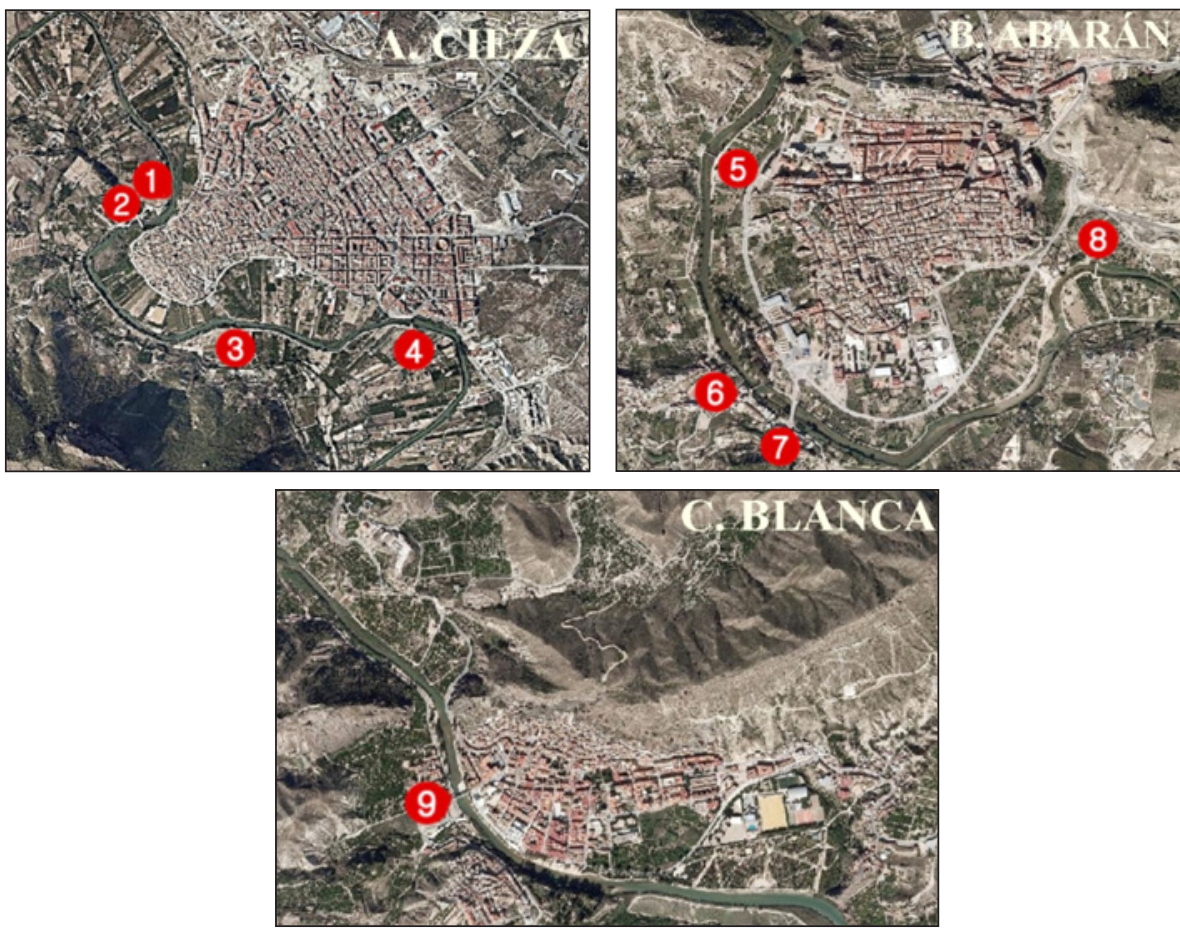

Fuente: Sitmurcia. Infraestructura de Datos Espaciales de la Región de Murcia.

Tabla 1

DATOS GENERALES Y ACTUALES DE LOS PUENTES HIDRÁULICOS

\begin{tabular}{|l|l|l|l|}
\hline \multicolumn{1}{|c|}{ PUENTES } & MUNICIPIO & \multicolumn{1}{c|}{ SERVICIO } & \multicolumn{1}{c|}{ ÉPOCA } \\
\hline Pasarela $\left(\mathrm{N}^{\circ} 1\right)$ & Cieza & Tráfico peatonal & Coetáneo \\
\hline Puente de los nueve ojos $\left(\mathrm{N}^{\circ} 2\right)$ & Cieza & Tráfico automóviles y peatonal & Edad Moderna \\
\hline Puente de Alambre $\left(\mathrm{N}^{\circ} 3\right)$ & Cieza & Tráfico peatonal & Desconoce \\
\hline Puente $\left(\mathrm{N}^{\circ} 4\right)$ & Cieza & Tráfico automóviles & Coetáneo \\
\hline Pasarela $\left(\mathrm{N}^{\circ} 5\right)$ & Abarán & Tráfico peatonal & Coetáneo \\
\hline Puente viejo $\left(\mathrm{N}^{\circ}\right.$ 6) & Abarán & Tráfico peatonal & Edad Moderna \\
\hline Puente nuevo $\left(\mathrm{N}^{\circ} 7\right)$ & Abarán & Tráfico automóviles y peatonal & Coetáneo \\
\hline Puente $\left(\mathrm{N}^{\circ} 8\right)$ & Abarán & Tráfico automóviles y peatonal & Coetáneo \\
\hline Puente de Hierro $\left(\mathrm{N}^{\circ} 8\right)$ & Blanca & Tráfico automóviles y peatonal & Edad Moderna \\
\hline
\end{tabular}

Fuente: Señalados su ubicación en la Figura 2. (Elaboración propia). 
por la doble rasante. Este modelo de edificación surgió de Juan Gómez. En cambio, el movimiento innovador estuvo representado por Melchor Luzón (Segado, 1990), Pedro y Gaspar de la Peña. Sus puentes eran cortos con rasante horizontal acompañados por pocos arcos de medio punto de amplia luz y situados altos, incluyendo un reforzamiento de las riberas (Redondo y Aramburu, 1996). Durante el siglo XVIII este tipo de estructuras comunicativas progresaron en su configuración gracias a la especialización de diferentes arquitectos e ingenieros, como por ejemplo Pedro de Ribera (1719) o el mismo Padre Pontones (Cano, 2004).

\section{Figura 3}

\section{FOTOGRAFÍAS ANTIGUAS DEL PUENTE DE BLANCA Y EL PUENTE DE LOS NUEVE OJOS DE CIEZA}
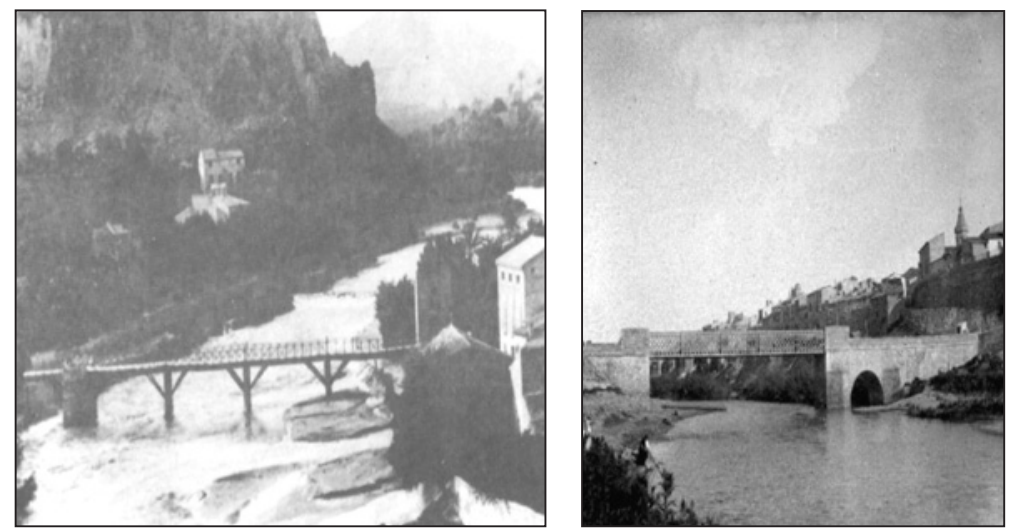

Fuente: Páginas webs detalladas a pie de página. $\mathrm{Blanca}^{3}$ y $\mathrm{Cieza}^{4}$.

La actual herencia patrimonial recibida de estos puentes en el área de investigación ofrece una escasa riqueza histórica material. El motivo de esta situación se explica a través de las destrucciones provocadas por las temibles riadas $\mathrm{y}$, especialmente, por la influencia evolutiva de la tecnología emergida en una etapa concreta. En efecto, las sucesivas intervenciones, las catástrofes naturales ocultan las huellas vetustas de estas fábricas, construidas en los mismos sectores durante casi los últimos seis siglos. Sin embargo, esta circunstancia no significa que no exista una documentación rica y relevante sobre estas unidades de ingeniería hidráulica, donde es posible observar detalles como la organización para edificar el edificio, como así los maestros y los órganos instituciones encargados de ello.

3 http://amigosdelosriosmurcianos.blogspot.com.es/2011/04/historia-del-puente-de-hierro-de-blanca.html Visto: $10-03-2017$

4 https://archivoweb.carm.es/archivoGeneral/arg.muestra_detalle?idses=0\&pref_id=4390922 Visto: 10-03-2017 


\section{LOS BIENES DE COMUNICACIÓN EN LA VEGA ALTA DEL SEGURA}

Los datos recogidos en la Tabla 2 representan toda aquella información que se ha podido extraer a través de las fuentes. Si son interpretados a fondo es posible dividirlos y clasificarlos en dos bloques generales: el primero centrado en vías de comunicación más rústicas, como son las cunas y barcos; y, en segundo lugar, otras de carácter más progresista, los puentes de madera y los puentes de piedra.

\section{Tabla 2 \\ LAS INFRAESTRUCTURAS DE COMUNICACIÓN HIDRÁULICA EN CIEZA, ABARÁN Y BLANCA (1500-1800)}

\begin{tabular}{|c|c|c|c|c|}
\hline AÑO & BIEN INMUEBLE & POBLACIÓN & ACTUACIÓN & PRECIO \\
\hline $1511^{5}$ & Barco & Cieza & Obra nueva & - \\
\hline $1515^{6}$ & Puente de Madera & Cieza & Obra nueva & - \\
\hline $1635^{7}$ & Puente de Madera & Cieza & Aderezó en el puente & 1.600 reales $^{8}$ \\
\hline $1675^{9}$ & Barco & Cieza & Obra nueva & 1.400 reales $^{-1}$ \\
\hline $1676^{10}$ & Cuna & Cieza & Obra nueva & - \\
\hline $1678^{11}$ & Barco & Cieza & - & - \\
\hline $1678^{12}$ & Puente de madera & Cieza & Obra nueva & - \\
\hline $1681^{13}$ & Puente de madera & Cieza & Obra nueva & 1.2000 reales \\
\hline $1681^{14}$ & Puente de Madera & Cieza & Aderezo del puente & 327 reales \\
\hline $1684^{15}$ & Puente de Madera & Cieza & Obra nueva & - \\
\hline $1688^{16}$ & Puente de Madera & Cieza & Reforma & 1.000 reales \\
\hline
\end{tabular}

5 Archivo General de Murcia, código digital: ES.30030.AGRM/FR,3 / Archivo Histórico Nacional > FR,3.2. / Órdenes Militares > FR,3.2.6. / Libros Manuscritos> FR,3.2.6.1 / Libros Manuscritos de la Orden de Santiago > FR,AHN,R-2/2 / Libro de visitas de las villas del partido de Murcia.

6 MARÍn CANO, A. (2010): Hierro, Piedra y Madera. Los puente de Ciezanos sobre el Segura (Siglos $X V I-X X)$. Cieza, Asociación Cultural Visigoda, pp. 21.

7 ARCHIVO MUNICIPAL DE CIEZA, de aquí hacia delante (A.M.C). Actas capitulares de 1609-1623. Sin título. 11-03-1635. Pág. 77

8 MARÍN CANO, A. (2010): Hierro, Piedra y Madera. Los puente de Ciezanos sobre el Segura (Siglos XVI-XX). Cieza, Asociación Cultural Visigoda, pp. 22.

9 ARCHIVO HISTÓRICO PROVINCIAL DE MURCIA, de aquí hacia delante A.H.P.M. NOTARIO, 9509. Don Andrés de Molina carta de pago a Francisco Jiménez. 24-02-1675. Pág. 34 recto - 34 vuelto.

10 A.M.C. Actas capitulares de 1673-1678. Decreto para visita de los mojones, 8-04-1676. Sin número de pág.

11 A.M.C. Actas capitulares de 1673-1678. Decreto de esta villa. 28.03-1678. Sin número de pág.

12 A.M.C. Actas capitulares de 1673-1678. Decreto de esta villa. 28.03-1678. Sin número de pág.

13 A.H.P.M.NOT, 9454. Escritura de contrato entre Don Miguel Marín Talón Alférez mayor y Joseph Marfil para hacer el puente. 22-02-1681. Págs. 27 recto - 27 vuelto

14 A.H.P.M.NOT, 9454. Carta de pago de Don Joseph Marfil maestro que ha venido al aderezo del puente a Doon Manuel Marín Talón. 25-09-1681. Págs. 95 recto - 95 vuelto

15 A.M.C. Actas capitulares de 1683-1689. Sin título. 22-10-1684. Sin número de pág.

16 A.M.C. Actas capitulares de 1683-1689. Sin título. 26-09-1688. Sin número de pág. 


\begin{tabular}{|c|c|c|c|c|}
\hline AÑO & BIEN INMUEBLE & POBLACIÓN & ACTUACIÓN & PRECIO \\
\hline $1748^{17}$ & Puente de Madera & Cieza & Obra nueva & - \\
\hline $1752^{18}$ & 4 puentes de Madera & Cieza & Obra nueva & 6.000 reales \\
\hline $1754^{19}$ & Puente de Piedra & Cieza & Obra nueva & 11.000 reales \\
\hline $1758^{20}$ & Barco & Cieza & Obra nueva & - \\
\hline $1759^{21}$ & Puente de Madera & Cieza & Obra nueva & - \\
\hline $1764^{22}$ & Puente de Madera & Cieza & Obra nueva & - \\
\hline $1773^{23}$ & Puente de Madera & Cieza & Reforma. Aderezo & 2.000 reales \\
\hline $1773^{24}$ & Puente de Madera & Cieza & Reforma. Madera & 1.400 reales \\
\hline $1776^{25}$ & Puente de Madera & Cieza & - & 6,598 reales \\
\hline $1782^{26}$ & Puente de Piedra & Cieza & Obra nueva & 35.000 maravedíes \\
\hline $1783^{27}$ & Puente de Madera & Blanca & Obra nueva & - \\
\hline $1792^{28}$ & Puente de piedra & Abaran & Obra nueva & - \\
\hline
\end{tabular}

Fuente: Archivos documentales y fuentes secundarias. Citas a pie de página (Elaboración propia).

\subsection{Comunicaciones hidráulicas rústicas: cunas y barcos}

Las cunas y los barcos fueron las unidades más habituales dentro de los modelos de comunicación hidráulica rural durante el periodo moderno, ya que también era costumbre de la época utilizar otro género estructural: los puentes de barcas unidas sobre las que se colocaban tablas (Arciniega, 2015). Las cunas se trataban de una vía de comunicación compuesta por una estructura alargada, que cruzaba de un lado a otro el río y estaba formada por dos maromas de esparto paralelas y listones de madera trasversales (Véase Ilustración I); los barcos de madera, por el contrario, se identificaban como una embarcación de estructura cóncava o aplanada que podía variar en sus dimensiones, dependiendo siempre de la adquisición económica disponible para liquidar todo su coste. Tales estructuras ofrecieron unas ventajas. Sobresalen: la rapidez de su construcción, la asequibilidad del precio con arreglo al material de construcción (madera, maromas, etc.).

17 A.M.C. Actas capitulares de 1745-1749. Decreto del 10 de diciembre 1748. 10-12-1748. Sin número de pág.

18 A.M.C. Actas capitulares de 1750-1754. Decreto 12 de noviembre. 12-11-1752. Sin número pág.

19 A.M.C. Actas capitulares de 1750-1754. Decreto de 26 de noviembre. 26-11-1754. Sin número de pág.

20 A.M.C. Actas capitulares de 1754-1759. Decreto sobre puente. 30-12-1758. Sin número de pág.

21 A.M.C. Actas capitulares de 1754-1759. Valga para el Reinado de su Majestad Don Carlos III. 1759. Pág. 36 recto.

22 A.M.C. Actas capitulares de 1760-1764. Decreto del 25 de noviembre. 25-09-1764. Sin número pág.

23 A.M.C. Actas capitulares de 1770-1774. Decreto del 17 de agosto. 17-08-1773. Sin número de pág.

24 A.M.C. Actas capitulares de 1770-1774. Decreto del 17 de agosto. 17-08-1773. Sin número de pág.

25 A.M.C. Actas capitulares de 1775-1780. Decreto. 20-05-1777. Sin número de pág.

26 A.M.C. Actas capitulares de 1780-1784. Decreto de 19 de Abril. 19-04-1782. Sin número de pág.

27 A.H.P.M. NOT, 9358. Poder de la justicia y ayuntamiento de Blanca a Madrid. 8-10-1778 Págs. 51 recto -52 recto.

28 A.H.P.M. NOT,9308. Venta Real Bartolomé de Lova a Juan Antonio Quílez. 10-03-1792 Págs. 9 recto 10 recto 
Los inconvenientes de estas infraestructuras residían en la condición precaria e inestable de su estructura, luego insegura, por la limitación tanto del personal que podía emplearlo al mismo instante, como la capacidad de peso que podía soportar, e, incluso, el escaso intervalo temporal que duraba en funcionamiento por la debilidad y fragilidad misma del material Esta característica última es posible comprobarla a través de la Tabla II, entre el año de 1675 y 1678.

Las poblaciones de Abarán y Blanca emplearon este tipo de unidades hasta las décadas finales del siglo XVIII. En los lugares donde estuvieron implantados los topónimos, tanto en Blanca como en Abarán, como "cuna" se asentaron los pilares, las vigas, los cimientos de unos puentes de madera y de piedra. Cieza, asimismo, utilizó este tipo de infraestructuras, pero más que cómo una funcionalidad y un medio práctico y duradero, fue con el objetivo de solventar velozmente los problemas de paso de los vecinos. Un ejemplo de este último comentario se representa en dos periodos: uno con la Orden de Santiago en 1515, cuando los visitadores santiaguistas decretan realizar un barco para ejecutar la moliente al molino harinero de río (Molino del Cebolla) que se encontraba en la vertiente opuesta a la localidad; y otro en 1758, debido al hundimiento y derrumbe del puente de madera, para resolver las necesidades de los vecinos y de los transeúntes.

Los barcos utilizados en Cieza como servicio de paso, sin contar los empleados por el propio Heredamiento de la Andelma cuando no había medio para transcurrir de un lado a otro del Segura, siempre estuvieron respaldados administrativamente por el concejo de la villa. La financiación de estas se efectuaban con el importe cobrado para pasar de un lado a otro hasta que fuera liquidado el precio total de la construcción. No obstante, eran edificados y restaurados por un maestro carpintero elegido por el encargado de gestionar la obra y, a su vez, delegado del concejo, conocido con el apelativo de comisario. Una muestra de lo que se viene refiriéndose fue cuando al carpintero, Francisco Jiménez, se le encomendó por parte del concejo de Cieza (Comisario, Andrés Molina) un barco con un coste de 1.400 reales en 1675 (Véase Tabla 2).

\subsection{Vía de comunicación hidráulica urbanística: los puentes de madera y de piedra}

Las fábricas de comunicación hidráulicas de carácter civil sobresalen por dos tipologías afines al material: unas realizadas solamente con madera, y otras constituidas entre piedras labradas por maestros picapedreros y madera. Estas tipologías de arquitectura ofrecieron a la comunidad local una confortable circulación. Pueden localizarse una hilada de ventajas y de inconvenientes. Entre las ventajas sobresale la mayor facilidad y la gran variedad en el género de tránsito (personas, carruajes, ganado). Por consiguiente, el rendimiento y la funcionalidad del inmueble de ingeniería era de mayor consideración, ya que portaba una faceta asistencial, de prestación a la sociedad en el servicio personal, laboral y comercial. Asimismo, se caracterizó por ser más perdurable en el tiempo, con una mayor estabilidad. Por tanto, obviaba tragedias y transmitía seguridad aquellos que lo utilizaban asiduamente.

Existen dos acontecimientos históricos antecedentes al Puente Viejo de Abarán, conocido como "Puente de las Herrerías", que aclaran la necesidad de su instalación por las ventajas comentadas anteriormente: a) Un ejemplo de la necesidad de paso de un lado a otro lo muestra el manifestó del presbítero de la Iglesia Parroquial de Abarán, Pablo Yelo 
Gómez, que no podía acudir a sus haciendas en el partido del Darrax (al otro lado del río) por no haber puente por donde pasar. ${ }^{29}$ b) La instalación del puente también supuso obviar tragedias, como sucedió con el fallecimiento de Mateo Martínez por ir a buscar leña al otro lado del río. Éste individuo cruzó el río con sus dos bestias menores, una de ellas estuvo en peligro de ahogarse y por socorrerla conllevó el riesgo de ahogarse en 1780. Al cabo de unos días enfermó y falleció ${ }^{30}$ Sí hubiera habido puente la defunción se hubiera remediado. Estos dos acontecimientos influenciaron a proyectar por parte del concejo de Abarán un puente de piedra a finales de siglo.

Por otra parte poseían inconvenientes como la tardanza en realizarse, la fragilidad y la escasa resistencia que ofrecían ante las riadas del Segura, los altos costes de las obras, a pesar de que los puentes de madera eran bastantes más asequibles en los presupuestos que los de piedra. Esto se explica por los materiales empleados, su reutilización y la mano de obra. Un ejemplo que respalda esta aserción se revela con la edificación de dos viaductos de madera. Uno construido en 1681 por José Marfil con un coste de 1.200 reales, y el segundo alzado en 1757 con 6.00 reales. Estos precios contrastan con los importe a pagar en los dos puentes de piedra edificados en 1754 y 1782, con un precio el primero de ellos por 13.000 reales y el segundo con un importe de 35.000 reales; casi veinte veces más que el puente de madera construido en 1681. Los puentes de piedra eran más consistentes por sus cimientos, por su mayor peso. Cieza en menos de tres años construyó su primer puente de piedra por manos de un arquitecto de Murcia, Pedro Pagan en 1754, a diferencia de la ciudad de Murcia que tardó casi 34 años (1700-1734) en erigir su puente. Este último suceso se explica, ora por la búsqueda de un ingeniero que asegurara la duración del puente hasta un largo plazo, ora por la falta de recursos económicos para invertir en una obra de gran capital o simplemente por sustitución, renuncia del proyecto a ejecutar (Hernández, 1976).

Por otro lado, la mayor amenaza de estas infraestructuras civiles era cuando se producía una crecida del río, hecho natural distintivo en la Edad Moderna en la cuenca del Segura (Navarro y Montaner, 1978-1979). Este fenómeno natural afectaba directamente a la estructura física del puente. Daba igual los materiales pesados, la consistencia de la cimentación, el sustento de las vigas. La madre naturaleza cuando se propiciaba a dar agua al Segura, éste, en plena furia, arremetía con todo a su paso. Un ejemplo muy clarificador fue, cuando tres años después de la construcción del puente de piedra en 1754, la fábrica quebró su estructura por una temible riada. Ahora bien, en un periodo de casi 280 años, desde el siglo XVI hasta el siglo XVIII, se obraron diez puentes de madera, dos puentes de piedra, cuatro barcos y una cuna en la localidad de Cieza, sin contar las intervenciones de reforma realizadas en sus diversas partes configurativas. Esta información verifica la variabilidad de las operaciones que tuvieron que realizarse, e, igualmente, esclarece las políticas económicas amoldadas y practicadas por el concejo local de esta población. No obstante, ¿Cuáles fueron los agentes que impulsaron esta constante actividad? ¿Existieron otros factores, además, de la necesidad del hacendado por lo que la villa de Cieza se implicó como una mayor inversión en este aspecto de servicio urbano? ¿Era tanta la necesidad del agricultor?

29 A.H.P.M. NOT,9307. Sin título. 1790. Págs. 149 recto -149 vuelto.

30 A.H.P.M. NOT, 9311. Testamento María Tornero Gómez. 1780. Sin número de pág. 
La primera cuestión se responde por medio de la práctica agrícola que desarrollaron cada villa. En lo que respecta a Cieza el terreno donde adquiría mayor productividad de frutos se localizaba en el otro lado del Segura a través de dos acequias, la primera y más antigua el Heredamiento de la Acequia de la Andelma, y, por otro lado, el Heredamiento de la Acequia de Don Gonzalo. En cambio, las huertas de regadío de las poblaciones de Abarán y Blanca estaban articulas a los pies de los núcleos urbanos; es decir, entre el río y las poblaciones. No se expandió un sistema de regadío continuo de gran extensión hasta la cuarta década del siglo XVIII en la vertiente contraria. Este motivo justifica y confirma la instalación de puentes en Abarán y Blanca años más tarde. No obstante, a pesar de la importancia económica local que poseía la agricultura para la supervivencia y manutención del habitante, participaron otros condicionantes para que la localidad de Cieza desarrollara este programa de operaciones. Los agentes fueron la orografía, la posición estratégica donde se encontraba los municipios en el territorio y la evolución demográfica de cada población.

\subsubsection{Orografía y asentamiento}

Cieza, a diferencia, de Blanca y Abarán se hallaba en un lugar de mayor abertura y comunicación. No estaba rodeada de una cadena montañosa y el acceso a ella era sencillo. Gracias a este rasgo del relieve, el territorio de Cieza y su puente fue el eslabón de unión entre el Noroeste y el Centro del Reino de Murcia. En su territorio se hallaba el camino real. Esta travesía comunicaba el Reino de Granada con el Reino de Murcia; vía, además, primordial para las poblaciones de Caravaca, Moratalla, Mula, Ricote, Cehegín, Calasparra, Pliego; lo que facilitaba la comercialización entre unos sectores territoriales y otros. Además de la actividad mercantil, el puente proporcionó facilidades para el tránsito humano, ya fuera por movimientos emigratorios u otros aspectos más personales. A estas dos justificaciones se debe añadir la trascendencia que supuso con el envió y la distribución de postas. Luego, es entendible que las edificaciones y las intervenciones fueran urgentes por pura necesidad social y comercial, ya no solo local, sino de un modo más global. Por el contrario, esta circunstancia no la presentaba los municipios de Blanca y Abarán. Su emplazamiento era más complicado por localizarse en el epicentro de un valle y en un relieve bastante escabroso, lo que dificultaba la circulación de cualquier individuo interesado en recorrerlo.

\subsubsection{Demografía}

Con el mismo rango de importancia, otro agente que influenció a Cieza para efectuar este programa continuo de operaciones fue originado y determinado por el aumento constante de su demografía (Sancho, 2004). La cifra de habitantes fue incrementando siglo tras siglo. Con el crecimiento poblacional provino una mayor demanda en recursos naturales y de alimentos, un incremento dinámico social (mercantil, comercial, laboral). Esta situación entrometió de lleno al concejo, ya que, como promotor económico y administrativo de la zona, estuvo obligado a ofrecer una serie de servicios urbanos. Entre estos se encontraban: facilitar la vida al poblador con pasar al otro lado del río. Como antagonismo a este 
panorama, se encuentran Blanca y Abarán, poblaciones con un gran descenso demográfico (expulsión de los moriscos y epidemias) durante el siglo XVII y en las que se acrecentó la demografía de nuevo durante el siglo XVIII (García Avilés, 2000). Si comparamos, la primera fecha en la que se data un puente de madera de Cieza (1615) con las fechas que se obtiene del primer puente de madera de Blanca (1776) y el puente de piedra de Abarán (1792), puede observarse que el nivel de demográfico es casi el mismo en Cieza en la fecha de 1615 que las de Abarán y Blanca en los últimas décadas del siglo XVIII.

\section{Tabla 3}

\section{EVOLUCIÓN DEMOGRÁFICA (1507-1797)}

\begin{tabular}{lccccccccccc}
\hline Años & $\mathbf{1 5 0 7}$ & $\mathbf{1 5 1 1}$ & $\mathbf{1 5 1 5}$ & $\mathbf{1 5 3 5}$ & $\mathbf{1 5 9 1}$ & $\mathbf{1 6 9 4}$ & $\mathbf{1 7 1 7}$ & $\mathbf{1 7 5 5}$ & $\mathbf{1 7 6 9}$ & $\mathbf{1 7 8 7}$ & $\mathbf{1 7 9 7}$ \\
\hline Cieza & 765 & 585 & 832 & 1566 & 2160 & 2160 & 2448 & 4179 & 4769 & 5582 & 5294 \\
Abarán & 130 & 180 & 135 & 292 & 544 & 340 & 292 & 916 & 896 & 905 & 1024 \\
Blanca & 387 & 360 & 360 & 679 & 812 & 456 & 428 & 996 & 1101 & 1357 & 1460 \\
\hline
\end{tabular}

Fuente: García Avilés, 2000, y Sancho, 2004, op.cit.

El 90\% de las veces el importe de estos proyectos, ya fuera de madera o de piedra, era contribuido por los vecinos de la villa a través del repartimiento; es decir, cada habitante de la villa debía contribuir con un impuesto. Esta tasa era igual para todos los vecinos. Cuando había escaso número de habitantes, el coste por cabeza era entonces más elevado, por lo que se le hacía más dificultoso al vecino contribuirlo. Este panorama coincidiría con la limitación poblacional y económica de Blanca y Abarán durante gran parte de la Edad Moderna. Esta fórmula, con rasgos muy definidos por la densidad demográfica, demuestra que el impacto en el paisaje urbanístico fluye de la densidad pobladora del núcleo poblacional. La exigencia de la construcción era por solucionar ciertas carencias sociales afines a las necesidades laborales, comerciales o personales. Si la población se localizaba en un estado decadente y mísero, debía de conformarse con un elemento rústico como sucede con la cuna en Abarán y en Blanca.

\subsubsection{Los procedimientos administrativos}

Si bien la demografía y la orografía determinaron la edificación de los puentes en Cieza, Abarán y Blanca, también hay que señalar que un organismo principal de estos núcleos urbanos, provocó su constitución, ya fuera para prosperar las instalaciones urbanas, progresar el acceso al territorio y evitar ciertos problemas sociales: los concejos locales.

Estas instituciones eran las encargadas de tramitar aquellos inmuebles urbanos para servicios sociales. Las facultades eran distintas en cada concejo. Por ejemplo, en Cieza para tratar y gestionar tal asunto encomendaba y comisionaba la obra (intervención o construcción de la fábrica) a un miembro del propio concejo, ya fuera regidor o alférez. Este cargo era delegado por la autoridad superior; es decir, por la elección del conjunto del concejo local. La última voz de decisión la tenían el alcalde ordinario (1570-1668), el 
gobernador o alcaldes mayores (1668-1800). ${ }^{31}$ Para ello, se seleccionaba siempre un comisario con el propósito de direccionar e inspeccionar la obra, buscar la tasa más ahorrativa, los maestros, los materiales, entre otros aspectos. Por el contrario, los concejos locales de Blanca y Abarán tuvieron otros hábitos de proceder. En este último los encargados de administrar y supervisar la obra eran los propios alcaldes ordinarios; los maestros debían pujar por la obra ante el propio concejo municipal: por el contrario, en Blanca se focalizaba tal cargo en el célebre alcalde de aguas, puesto que era ocupado por la progenie de los Castillo. En este último caso, cuando sucedía cualquier imprevisto el alcalde de aguas informaba al concejo. Ahora bien, la subvención de la obra se dispuso por medio de cuatro vías financieras:

1. Prorrateo en la población local. Este método consistía en establecer un canon fijo para todo aquel ciudadano de la villa. Esto sucedió en Blanca en 1778 cuando solicita al concejo de las Órdenes un repartimiento entre toda la villa hasta alcanzar la cifra capital de 6.000 reales.

2. Repartimiento e importe contribuido por otras villas por la utilización del puente. Este procedimiento reside en una financiación ofrecida por cada villa que utilizase el servicio del puente. Un ejemplo de este caso sucede cuando en 1678 el concejo local de Cieza solicita a las villas de Cehegín, Moratalla, Pliego, Ricote, Ojos, Villanueva, Letur, Ferez, Socovos, Mula y Caravaca una cierta cantidad de dinero para la construcción del nuevo puente de madera. Tal petición se fundamentaba en la utilidad de la infraestructura, ya que formaba parte el puente del camino real hasta la ciudad de Murcia. También, además se debía por el tránsito del ganado. Los vecinos de Cieza en total contribuyeron a la obra con 2.000 reales de vellón. ${ }^{32}$

3. A través del caudal del pósito de la propia villa, como sucede en Cieza en 1684 cuando el rey, por medio de delegados, admitía que la obra fuera contribuida por el pósito de esta. ${ }^{33}$

4. Como medio de donación por un propio benefactor altruista, así se muestra en Abarán para la construcción del puente en 1795 con una donativo de 3.860 reales al caudal de la construcción de la fábrica por parte de Roque Yelo Molina. ${ }^{34}$

A parte del personal directivo de la empresa, el encargado de dirigir la contabilidad era el mayordomo del pósito del concejo municipal. También era conocido como "depositario". Para que la cifra total del proyecto no sobrepasara los presupuestos esperadas solicitaban a los vecinos ser participes como peones en la obra, como también en la ayuda del

31 En 1668 se creó, por decisión de Carlos II, el Partido Judicial de Cieza. Esta situación se explica por una cadena de altercados que se cometieron en dicha población. La constitución de este órgano, encabezada por la elección de un miembro santiaguista (Orden de Santiago) como gobernador y un alcalde mayores. La entrada de estos miembros supuso la desaparición del alcalde ordinario en el concejo local de Cieza hasta al menos principios del siglo XIX.

32 A.H.P.MU.NOT, 9511. Poder del ayuntamiento de esta villa a Alonso Fernández Quijada. 28-03-1678 Pág. 34 recto-36 vuelto

33 A.M.C. Actas capitulares de 1683-1689. Sin título. 22-10-1684. Sin número de págs.

34 A.H.P.MU.NOT, 9308. Dación en Pago, Roque Yelo Molina a Pasqual Rodríguez Fernández. 1795. Pág. 35 recto- 35 vuelto 
transporte de materiales. Esta circunstancia acontece en 1638, cuando el comisario Pedro López, alférez de Cieza, intentó la colaboración de todo el pueblo en la obra del puente de madera, además, añadiendo a esta actividad la proporción del repartimiento por cabeza vecinal de 8 reales. Luego, el vecindario, además de afrontar las cuentas, estaba obligado a prestar sus servicios al concejo, con el fin de rebajar el importe total de la construcción.

\subsubsection{Personal involucrado y organización laboral en las edificaciones}

Para realizar estas edificaciones era necesario doctos en la materia constructiva, ya fuera en el campo de carpintería, de la arquitectura, de la ingeniería o como maestro de puentes. Estos profesionales se encargaron de proyectar, diseñar, construir y mantener la arquitectura civil y comunicativa. Antes de implicarse en el ante proyecto, en el proyecto de ejecución y en la dirección de la obra, la empresa era subastada mediante pujas. El concejo, el comisario, el alcalde ordinario o el alcalde de aguas seleccionaba el pujador que más beneficiaba a la comunidad; es decir, el que ofrecía menor coste y el que asegurara unas garantías positivas para el levantamiento y la estructura del inmueble. El maestro antes de actuar directamente con el asentamiento del puente consideraba y valoraba el mejor emplazamiento para su instalación.

Buscaba las siguientes condiciones: Seguridad de estabilidad de la tierra y fluvial, donde el cauce debía ser de gran capacidad para que no fuera fácilmente desbordable con las lluvias torrenciales; lugares de cauce estrecho, se buscaba estos sitios para reducir y ahorrar en gastos; emplazamiento rectilíneo, antes de una curva, debido a la erosión lateral y a la erosión del fondo; ubicación próxima a los núcleos urbanos; la instalación en un terreno elevado para evitar el contacto de las aguas fluviales, ya que mermaban la solidez de la fábricas de paso.

Tabla 4

MAESTROS QUE INTERVINIERON EN LA ELABORACIÓN DE BARCOS Y PUENTES

\begin{tabular}{|l|l|c|l|l|}
\hline \multicolumn{1}{|c|}{ MAESTROS } & $\begin{array}{l}\text { TÉCNICA. } \\
\text { LOCALIDAD }\end{array}$ & AÑO & OBRA Y REFORMA & \multicolumn{1}{|c|}{ PUENTES } \\
\hline Juan Gómez & $\begin{array}{l}\text { Carpintero } \\
\text { (Cieza) }\end{array}$ & 1615 & $\begin{array}{l}\text { Cortar madera para } \\
\text { reparar el puente }\end{array}$ & $\begin{array}{l}\text { Puente de Madera } \\
\text { (Cieza) }\end{array}$ \\
\hline $\begin{array}{l}\text { Francisco } \\
\text { Jiménez }\end{array}$ & Carpintero & 1675 & Fabricación de un barco & Barco nuevo (Cieza) \\
\hline José Marfil & Carpintero & 1681 & $\begin{array}{l}\text { Construcción de un } \\
\text { puente de madera nuevo }\end{array}$ & $\begin{array}{l}\text { Puente de Madera } \\
\text { Nuevo (Cieza) }\end{array}$ \\
\hline José Marfil & Carpintero & 1681 & $\begin{array}{l}\text { Aderezo del puente de } \\
\text { madera }\end{array}$ & $\begin{array}{l}\text { Puente de madera } \\
\text { (Cieza) }\end{array}$ \\
\hline Juan Pagán & $\begin{array}{l}\text { Maestro y } \\
\text { arquitecto de } \\
\text { Murcia }\end{array}$ & 1754 & $\begin{array}{l}\text { Dirección, planificación } \\
\text { de nuevo puente de } \\
\text { piedra }\end{array}$ & $\begin{array}{l}\text { Puente de piedra } \\
\text { (Cieza) }\end{array}$ \\
\hline
\end{tabular}




\begin{tabular}{|c|c|c|c|c|}
\hline MAESTROS & $\begin{array}{l}\text { TÉCNICA. } \\
\text { LOCALIDAD }\end{array}$ & AÑ̃ & OBRA Y REFORMA & PUENTES \\
\hline $\begin{array}{l}\text { Bartolomé } \\
\text { Rodríguez }\end{array}$ & $\begin{array}{l}\text { Picapedrero y } \\
\text { cantero }\end{array}$ & 1772 & \multirow{2}{*}{$\begin{array}{l}\text { Suministro de } 200 \text { varas } \\
\text { de piedra labrada al } \\
\text { precio de } 11 \text { reales por } \\
\text { una }\end{array}$} & \multirow{2}{*}{$\begin{array}{l}\text { Puente de Piedra } \\
\text { (Cieza) }\end{array}$} \\
\hline $\begin{array}{l}\text { Alejandro de } \\
\text { Lastra }\end{array}$ & $\begin{array}{l}\text { Picapedrero y } \\
\text { cantero }\end{array}$ & 1772 & & \\
\hline $\begin{array}{l}\text { Lorenzo } \\
\text { Montellón }\end{array}$ & $\begin{array}{l}\text { Maestro de } \\
\text { puentes }\end{array}$ & 1776 & \multirow{2}{*}{$\begin{array}{l}\text { Dirección, planificación } \\
\text { de la nueva fábrica de } \\
\text { piedra }\end{array}$} & \multirow{2}{*}{$\begin{array}{l}\text { Puente de Piedra } \\
\text { (Cieza) }\end{array}$} \\
\hline $\begin{array}{l}\text { Francisco } \\
\text { Moreno }\end{array}$ & Alarife & 1776 & & \\
\hline Juan de Lastra & $\begin{array}{l}\text { Picapedrero y } \\
\text { cantero (Ricote) }\end{array}$ & 1783 & $\begin{array}{l}\text { Hilada de piedras de } \\
\text { sillería para poder } \\
\text { sostener las vigas }\end{array}$ & $\begin{array}{l}\text { Puente de madera } \\
\text { (Blanca) }\end{array}$ \\
\hline Gaspar Box & $\begin{array}{l}\text { Picapedrero } \\
\text { y Cantero } \\
\text { (Crevillente) }\end{array}$ & $1797^{35}$ & \multirow{2}{*}{$\begin{array}{l}\text { Dos hiladas del } \\
\text { escantillón del cuatro. } \\
\text { Tres de dos. } \\
\text { pilares de soga dos } \\
\text { palmos de espesor } \\
\text { Sillares de tisón tres } \\
\text { palmos de ancho }\end{array}$} & \multirow{2}{*}{$\begin{array}{l}\text { Puente de Piedra } \\
\text { (Abarán) }\end{array}$} \\
\hline $\begin{array}{l}\text { Manuel de } \\
\text { Cremadas }\end{array}$ & $\begin{array}{l}\text { Picapedrero } \\
\text { y Cantero } \\
\text { (Crevillente) }\end{array}$ & 1797 & & \\
\hline Juan Sánchez & $\begin{array}{l}\text { Arquitecto e } \\
\text { Ingeniero }\end{array}$ & 1797 & $\begin{array}{l}\text { Dirección para construir } \\
\text { puente de piedra }\end{array}$ & $\begin{array}{l}\text { Puente de piedra } \\
\text { (Abarán) }\end{array}$ \\
\hline Josef & $\begin{array}{l}\text { Alistador } \\
\text { (Abarán) }\end{array}$ & 1797 & $\begin{array}{l}\text { Supervisar la longitud y } \\
\text { la calidad del material }\end{array}$ & $\begin{array}{l}\text { Puente de piedra } \\
\text { (Abarán) }\end{array}$ \\
\hline
\end{tabular}

Fuente: Información extraída mayormente por las citas de la Tabla 3. (Elaboración propia)

Algunas de estas cinco condiciones del espacio coinciden en Cieza, Abarán y Blanca. Por ejemplo, en las cercanías o a los pies de las tres villas se ubicaron los puentes, como asimismo estos estuvieron articulados en un emplazamiento rectilíneo donde el cauce del rio era estrecho y profundo. Sin embargo, algo que favorece a las dos últimos municipios citados fue la altitud donde fueron instalados. En Abarán, por ejemplo, fue emplazado en un pequeño barranco, mientras que Blanca se favorecieron por la pendiente de la montaña donde estaba establecido el núcleo del pueblo. A pesar de que muestre la impresión que con la altura se evitaba los daños de las riadas, no era suficiente. Cuando el río sufría en su cauce una crecida no tenían casi utilidad alguna por los desperfectos ocasionados, y, de vez en cuando, se reutilizaban sus materiales.

Estos maestros estaban acompañados por sus cuadrillas. Tales grupos se componían con diferentes trabajadores. Se encontraban los especialistas (carpinteros y canteros) de proporcionar y moldear los materiales para la obra. El maestro era el encargado de dictaminar las directrices (dimensiones, cantidad) de los elementos constituyentes de la obra

35 A H.P.M. NOT, 9310. Real Obligación de Manuel Cremades y Gaspar Box, con los concejos de la villa de Abarán, Reino de Murcia. 2-10-1795. Sin número de pág. 
y, además, de supervisarlos, como sucede en Abarán con los picapedreros de Crevillente en 1797. No obstante, los maestros que dirigían el proyecto tenían la posibilidad de contratar a obreros mayores como apoderados (representantes físicos que supervisaban y direccionaba a través de las pautas del maestro mayor la obra) o de menor calado para la reforma o construcción del puente. El primero de ellos era como el dirigente que visionaba y direccionaba la obra, cumpliendo con todas las directrices sugeridas por el maestro de puente, ingeniero o arquitecto. Esto sucede con el alarife Francisco Moreno en 1776 con la construcción del puente de piedra. Un ejemplo de obreros de menor calado se trata del alarife mayor. En 1681 ocho hombres participaron a expensas del maestro José Marfil con un salario de cinco reales al día para la construcción del puente de madera. Duraron veintiún días de trabajo, con una cantidad total de 840 reales $^{36}$. Igualmente la población podía colaborar en la obra, transportando el material de un lugar a otro con la ayuda de sus animales domésticos. Un patrón de este hecho sucede en 1775 cuando se calcula el ahorro por la elevada participación vecinal en 3.500 reales $^{37}$. Luego, para realizar la obra había como una cadena de cuerpos laborales, pendientes de la experiencia y los cometidos que disponía (Véase Tabla Jerárquica I).

\section{Tabla jerárquica 1 \\ GRADOS DE PODER Y ESCALA LABORAL}

$1^{\circ}$ Nivel

Maestros de puentes (Ingenieros, arquitectos, Carpinteros)

\} El regente

$2^{\circ}$ Nivel Alarife Mayor

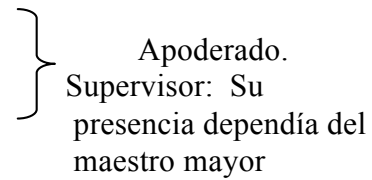

$3^{\circ}$ Nivel Maestros canteros ,carpintería , caleros

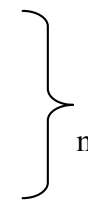

Individuos hábiles en

la elaboración de los materiales de construcción.

$4^{\circ}$ Nivel Obreros menores

$5^{\circ}$ Nivel Población

$6^{\circ}$ Nivel Bestias y animales

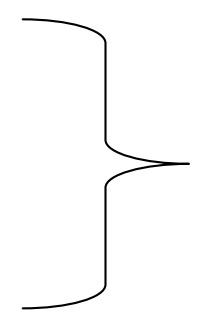

Peones, ayudantes, colaboradores

Participación directa en la fábrica

Fuente: Elaboración propia.

36 A.H.P.M. NOT, 9454. Carta de pago Joseph Marfil vecino de la ciudad de Murcia de Manuel Marín Talón Alférez mayor. 2-05-1681. Pág. 43 recto - 43 vuelto.

37 A.M.C. Actas capitulares de 1775-1780. Decreto. 20-05-1777. Sin número de págs. 
En lo que atañe a la extracción del material se buscaba el recurso lo más cercanamente posible. Esto se explica por la reducción de los costes y la agilidad y ligereza de trasportar al punto clave todo el material. Un claro ejemplo ocurre con la construcción del puente de piedra de Abarán, donde próximo a los cimientos de la fábrica estaba la extracción de piedra. Si la villa debía extraer el material en otra jurisdicción, como es entendible, debía contribuir por el consumo y aprovechamiento del recurso. Este caso sucede en Cieza por la falta de madera de buena calidad en sus montañas acudiendo a la jurisdicción de Hellín para la tala de pinos. En efecto, cuando el estado del puente de madera estaba deteriorado el concejo declaraba la urgencia de intervenir en él, ora por la escasa cantidad de madera que atesoraba la jurisdicción, ora para evitar mayores gastos en la construcción de una nueva fábrica ${ }^{38}$.

Igualmente el aderezo de los puentes de madera fue una manera de actuar en estas infraestructuras. La fuerza de las bestias se empleaban para remendar el equilibrio de la estructura a través del empuje de la propia bestia, tirando con maromas; o en el esqueleto del propio viaducto de madera se colocaban como un remedio técnico un arquetipo de piedras pesadas encima de las vigas para aumentar la resistencia de los cimientos con el propósito de que no retrocediera la estructura por la corriente del lecho fluvial. Tal técnica fue dispuesta en el puente de madera de Blanca con el encargo al maestro cantero Juan de Lastra, vecino de la villa de Ricote, de cierta cantidad de piedras labradas en 1783.

\section{LA TRASCENDENCIA DEL PAISAJE EN EL ESPECTADOR}

El entorno de los tres puentes de la Edad Moderna esclarece una interacción y una combinación entre la naturaleza y las actuaciones por distintas configuraciones del hombre a lo largo del tiempo en un territorio concreto. Su paisaje se entiende precisamente como la exposición visible y sensorial, la revelación física, del orden natural modelado e intervenido por los autóctonos. Tal panorama permite al espectador conocer, no solo el perfil orográfico de la zona, sino reconocer los integrantes del territorio, la disposición que lo justifica y su significado referente al ámbito natural como artificial. Por ejemplo, es innegable la trascendencia que toma el Río Segura para los habitantes de estas poblaciones. Es un lazo básico y esencial, la mayor identidad del área. Representa, sin duda alguna, a raíz de las acequias y las huertas en la cuenca fluvial de la Vega Alta del Segura la dependencia, tanto en el pasado como el presente, de un colectivo sobre este elemento natural. En efecto, este panorama incita a interpretar o a cuestionarse el vínculo del paisaje y las acciones y evoluciones históricas que lo han conducido hasta la actualidad. Desde la estructura de los puentes, todo lo que se viene hablando, puede experimentarse cognitivamente, e, incluso, inducir reacciones sensoriales mediante unos agentes, que catalogan y caracterizan al paisaje en distintas categorías a raíz de unas cualidades que forman parte de su unidad:

a) La sonoridad, lo aromático y las tonalidades de color. Los efectos que provoca el cauce fluvial es imprescindible para que el espectador conecte con el medio. El ritmo y el dinamismo de la corriente hídrica, junto a los sonidos de la flora por el

38 A.M.C. Actas capitulares de 1754-1759. Valga para el Reinado de su Majestad Don Carlos III. 1759. Págs. 36 recto 
movimiento del viento y los rumores de algunas aves, producen un escenario ideal, especialmente por las características que presenta el entorno del puente viejo de Abarán (Véase Figura 4). A ello hay que añadir la emanación húmeda que produce la esencia del Segura y la entremezcla de las tonalidades de color.

\section{Figura 4}

\section{VISTA DESDE EL PUENTE VIEJO DE ABARÁN}

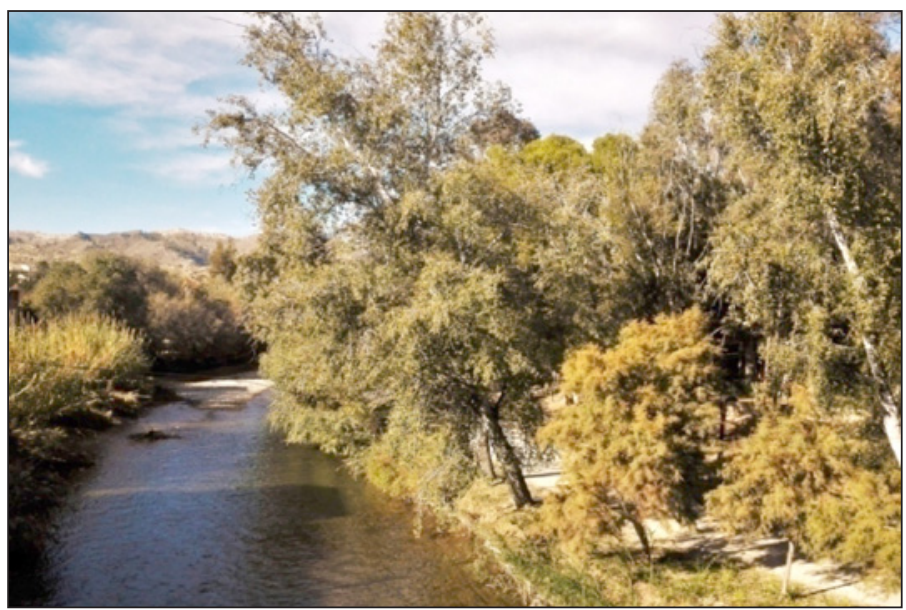

Fuente: Imagen optenida desde el Puente Viejo. (Fotógrafo J.C. Trigueros, 10/09/2017)

b) Hábitat natural donde es posible contemplar una variedad de especies. La flora puebla la ribera del río. Las especies más comunes son los álamos, los chopos, las aneas, las cañas. En referencia a la fauna se encuentra la nutria como animal carnívoro (YELO \& CALVO, 2004). Se nutre de carpas, cangrejos de rio, anfibios, barbos, entre otras especias. También resalta la presencia de garzas reales, de patos, la garceta común, de ruiseñores, de gorriones, del mirlo acuático, sin dejar olvidados los diferentes insectos localizados a lo largo de la ribera.

a) Lo orográfico. Este modelo, también basado en la visión, posibilita observar la articulación del relieve de la zona: vegas fluviales de reducido espacio a causa de la cercanía de cuerpos de tierra elevados a la ribera del río, pueblos incrustados en valles, como muestra los ejemplos de Abarán y Blanca, donde puede contemplarse fondos montañosos, entre otros aspectos.

b) La cultura y la historia. El puente de los nueve ojos y el puente de hierro de Blanca son los que muestran la mayor conexión con este paisaje afin a bienes inmuebles simbólicos para los habitantes de estas poblaciones. Desde el primero y puede percibirse el casco antiguo de Cieza (muro de la fortaleza antigua de la encomienda santiaguista, el campanario de la torre de la basílica de la Asunción, entre otros aspectos). Desde el segundo puede advertirse la fortificación musulmana de Blanca en la cumbre de una montaña (Figura 5). 
La visión panorámica ofrecida por los puentes brinda la oportunidad de percibir el género paisajístico existente y su interpretación en diferentes escalas. Su valor gracias al paisaje, no solo está pendiente de elementos tangibles o intangibles, sino también parte de la consideración de la calidad visual del entorno inmediato, de la calidad del fondo escénico y de la asiduidad humana. Por tanto, estas unidades evidencian un paisaje rico, activo y efectivo.

\section{Figura 5}

\section{VISTAS CULTURALES DESDE EL PUENTE DE HIERRO DE BLANCA Y EL PUENTE DE NUEVE OJOS DE CIEZA}

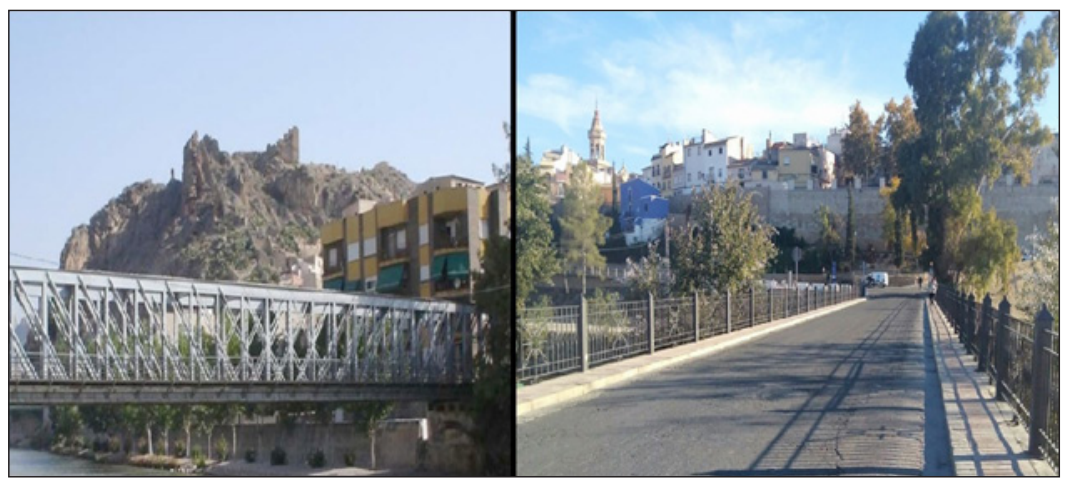

Fuente: Imagen obtenida junto al puente de Hierro de Blanca y el Puente de los Nueve Ojos en Cieza (Fotógrafo J.C. Trigueros, 10/09/2017).

\section{CONCLUSIONES}

Más allá de los aspectos físicos y visuales, la planificación del territorio turístico se trata de un instrumento esencial para impedir consecuencias negativas, como causar malestar al individuo interesado en la práctica turística por no haber una organización o perjudicar el ecosistema y el entorno. Luego, es necesaria una ordenación del material didáctico, como también de la actividad. La rentabilidad y la atracción deben principiarse a través de dos estados para el turista: el pasivo y de aprendizaje, y el activo y dinámico, como puntos de pesca (Puente Viejo de Abarán), prácticas artísticas, entre otras actividades.

Este último estado lleva a diseñar planes a seguir, por ejemplo, paneles explicativos para valorar el paisaje y la obra. Asimismo, es importante de que las empresas, que traten habitualmente con adultos, propongan un itinerario deportivo y didáctico. Comenzar en uno de los puentes, ya sea de Cieza o en Blanca, seguir el lecho fluvial por medio de una práctica deportiva (ciclismo, senderismo, etc.), ya que existen caminos rurales cerca del borde del Segura que unen a las distintas poblaciones, y detenerse en los puntos claves del itinerario: los puentes históricos. Ya en ellos es recomendable ofrecer, además de la información, otras actividades, como un concurso fotográfico con el fin de publicarlo en medios audiovisuales. En resumen, es un itinerario donde se combinan dos prácticas habituales en el turismo: la acción continua y la ilustración cognitiva. 
Ahora bien, del presente trabajo se han derivado los siguientes resultados: En primer lugar que los puentes de la Vega Alta del Segura son elementos atractivos para el desarrollo y actividad turística. Se encuentran en un espacio histórico de gran intensidad y actividad social. Por lo que son parajes a conservar. Otro punto ha sido el renovado conocimiento sobre estas unidades de comunicación, especialmente en el Puente Viejo de Abarán (BIC) y el Puente de Hierro de Blanca al rebajar las fechas de sus edificaciones, y al especificar eventos determinantes que hasta ahora se desconocían. Sobre el Puente de Nueve Ojos de Cieza, en cambio, la monografía de Marín Cano coincide con todos los documentos hallados, hasta el $90 \%$ de los datos acumulados sobre este bien inmueble. Sin embargo, ha sido necesario organizar en distintas líneas los testimonios, con arreglo a esclarecer las discrepancias y las semejanzas, y configurar un contenido didáctico y estructurado. Luego, a estas vías de comunicación se las debe considerar y analizar con detenimiento por dos motivos: Primeramente, porque conllevan valores patrimoniales y aportan contenidos culturales para el conocimiento humano; y, seguidamente, porque son un reflejo de insistencia, conservación y de esfuerzo de una población, al mantener el paraje y el medio vigente.

Igualmente ha sido comprobado no solo el valor pragmático y evolutivo de estas infraestructuras, sino el valor ambiental y paisajístico que las envuelven. Si bien, son componentes que con ciertas estrategias pueden servir de atracción para turistas e interesados. Luego, aporta una dualidad: un turismo contemplativo, basado en experiencias cognitivas y sensoriales; y un turismo activo. En definitiva, los puentes son bienes patrimoniales con valores atrayentes, que facilitaron, como ya se ha comentado, la fluidez social, la unión de espacios aislados, el impedimento de tragedias y el progreso en un determinado territorio.

\section{BIBLIOGRAFÍA}

ARCINIEGA, L. (2015): "Puentes de canteria en el Reino de Valencia de la Edad Moderna: Construcción y polisemia" . En Lexicon, Storia e architettura in Sicilia e nel Mediterrraneo. Ponti in pietra nel Mediterraneo en eta moderna, Palermo, Caracol, pp. 21-34.

BARRIO MOYA, J. L. (1986): "El hermano Francisco Bautista y la desaparecida facahada de la Catedral de Cuenca. Imafronte, $\mathrm{n}^{\circ}$ 2, pp. 57-64.

CANO SANZ, P. (2004): Fray Antonio de San José Pontones: arquitecto, ingeniero y tratadista en España, (1710-1774). Madrid, Universidad Complutense de Madrid. Tesis Doctoral.

CORRELA SUÁREZ, P. (1985): "El hermano Bautista y otros maestros en las obras de la Iglesia Parroquial de Navalcarnero durante los siglos XVII y XVIII". Anales del Instituto de Estudios Madrileños, n 22, pp. 81-95.

DÍAZ MORENO, F. (2008): Fray Lorenzo de San Nicolas. Madrid, Instituto de Estudios Madrileños .

FERNÁNDEZ DEL HOYO, M. A. (1994): “Una Obra de Fray Lorenzo de San Nicolas en Valladolid. En Tiempo y espacio en el arte: homenaje al profesor Antonio Bonet Corréa. Madrid, Universidad Complutense, pp. 481-488. 
GARCÍA AVILES, J.M. (2000): El Valle de Ricote: Fundamentos económicos de la encomienda santiaguista. Murcia, Real Academia Alfonso X el Sabio.

GARCIA MAYOR, C. y CANALES MARTÍNEZ, G. (2017): “Catral de la huerta tradicional a la huerta urbanizada. La transformación del paisaje agrario en el Bajo Segura: Del Animus Regandi al Animus Aedificandi”. Cuadernos de Turismo, n 39, pp. 191-213. HERNÁNDEZ ALBADALEJO, E. (1976): "El puente viejo de Murcia". Anales de la Universidad de Murcia. Filosofía y letras, vol. 34 (1), pp. 111-118.

LÓPEZ GAYARRE, P.A. (1990): Fuentes bibliográficas de arte y uso de arquitectura de Fray Lorenzo de San Nicolas. Espacio, tiempo y forma. Serie VII. Historia del Arte, vol. 3, pp. 137-150.

MARTÍNEZ GARCÍA, F. (2010): "El puente viejo de Abarán: historia y técnica". En I Jornada de Investigación y Divulgación sobre Abarán. Abarán, Asociación Carráhila, pp. 100-123.

NAVARRO HERVAS, F. y MONTANER SALAS, M.E. (1978-1979): “Inundaciones catastróficas, precipitaciones torrenciales y erosión en la provincia de Murcia”. Papeles del Departamento de Geografía, $\mathrm{n}^{\circ}$ 8, pp. 48-91.

REDONDO CANTERA, M. J. y ARAMBURU ZABALA, M.Á. (1996): La construcción de puentes en el siglo XVIII: innovacción y tradición. En Actas del Primer Congreso Nacional de Historia de la Construcción. Madrid 19-21 septiembre de 1996. Madrid, Instituto de Juan Herrera, pp. 435-443.

SÁNCHEZ BARIGA, A. y RODA SANCHO, J. (2001): "Fray Lorenzo de San Nicolas". Restauración \& Rehabilitación $n^{\circ} 48$, pp. 42.49.

SANCHO ALGUACIL, R. (2004): "Evolución demográfica de Cieza durante la Edad Moderna". En Historia de Cieza. Implantación, desarrollo e inicio de la disolución del sistema feudal en Cieza siglos XIII-XVIII.Murcia: Ayuntamiento de Cieza, vol. III, pp. 77-153.

SEGADO BRAVO, P. (1990): Melchor Luzón (Ingeniero, escultor, arquitecto, matemático y cosmógrafo) (1625-1698). Calamocha, Ayuntamiento de Calamocha.

YELO, N.D. y CALVO, J.F. (2004): “Distribución y estatus de los mamíferos carnivoros en la Región de Murcia”. Galemys, vol 16, n 2, pp. 21-37.

\section{WEBGRAFÍA}

http://amigosdelosriosmurcianos.blogspot.com/. Visto 07-08-2017

https://archivoweb.carm.es/archivoGeneral/arg.muestra_detalle?idses=0\&pref_ $\mathrm{id}=4390922$ Visto: $10-07-2017$

http://www.boe.es/buscar/doc.php?id=BOE-A-2006-3141 Visto: 12-12-2016 
